\title{
A Methodology for the Offline Evaluation of Recommender Systems in a User Interface with Multiple Carousels
}

\author{
Nicolò Felicioni \\ Politecnico di Milano, Italy \\ nicolo.felicioni@polimi.it
}

\author{
Maurizio Ferrari Dacrema \\ Politecnico di Milano, Italy \\ maurizio.ferrari@polimi.it
}

\author{
Paolo Cremonesi \\ Politecnico di Milano, Italy \\ paolo.cremonesi@polimi.it
}

\begin{abstract}
Many video-on-demand and music streaming services provide the user with a page consisting of several recommendation lists, i.e., widgets or swipeable carousels, each built with a specific criterion (e.g., most recent, TV series, etc.). Finding efficient strategies to select which carousels to display is an active research topic of great industrial interest. In this setting, the overall quality of the recommendations of a new algorithm cannot be assessed by measuring solely its individual recommendation quality. Rather, it should be evaluated in a context where other recommendation lists are already available, to account for how they complement each other. This is not considered by traditional offline evaluation protocols. Hence, we propose an offline evaluation protocol for a carousel setting in which the recommendation quality of a model is measured by how much it improves upon that of an already available set of carousels. We report experiments on publicly available datasets on the movie domain and notice that under a carousel setting the ranking of the algorithms change. In particular, when a SLIM carousel is available, matrix factorization models tend to be preferred, while item-based models are penalized. We also propose to extend ranking metrics to the two-dimensional carousel layout in order to account for a known position bias, i.e., users will not explore the lists sequentially, but rather concentrate on the top-left corner of the screen.
\end{abstract}

\section{CCS CONCEPTS}

- Information systems $\rightarrow$ Collaborative filtering; Recommender systems; • General and reference $\rightarrow$ Evaluation.

\section{KEYWORDS}

Recommender Systems; User Interface; Evaluation

\section{ACM Reference Format:}

Nicolò Felicioni, Maurizio Ferrari Dacrema, and Paolo Cremonesi. 2021. A Methodology for the Offline Evaluation of Recommender Systems in a User Interface with Multiple Carousels. In Adjunct Proceedings of the 29th ACM Conference on User Modeling, Adaptation and Personalization (UMAP '21 Adjunct), June 21-25, 2021, Utrecht, Netherlands. ACM, New York, NY, USA, 6 pages. https://doi.org/10.1145/3450614.3461680

Permission to make digital or hard copies of part or all of this work for personal or classroom use is granted without fee provided that copies are not made or distributed for profit or commercial advantage and that copies bear this notice and the full citation on the first page. Copyrights for third-party components of this work must be honored For all other uses, contact the owner/author(s).

UMAP '21 Adjunct, fune 21-25, 2021, Utrecht, Netherlands

(c) 2021 Copyright held by the owner/author(s).

ACM ISBN 978-1-4503-8367-7/21/06.

https://doi.org/10.1145/3450614.3461680

\section{INTRODUCTION}

Video on demand and music streaming services are among the most successful application domains of Recommender Systems. Often, in a video-on-demand service (e.g., Netflix, Amazon Prime Video) or on a music streaming platform (e.g., Spotify) the user is provided with multiple rows of recommendations, each generated according to a specific criterion, e.g., most recent, most popular, editorially curated (see Figure 1). These rows are referred to as widgets, shelves or as carousels. In this scenario, the user satisfaction and behavior does not depend on a single recommendation list but rather on the entire set of recommendations provided in the various carousels, as well as their position. Finding appropriate combinations of algorithms and ranking them to provide the user with a personalized page is an active research topic of significant industrial interest $[2,8,18]$.

Despite this, in the traditional offline evaluation scenario each recommendation model is evaluated independently and the one with the highest quality is preferred. This evaluation procedure does not take into account how would the different recommendation lists complement each other in a real carousel user interface. As a consequence, it may lead to the selection of algorithms that provide similar sets of recommendations. Since it is known that a set of diverse recommendations improves user satisfaction [3], and recommending the same item in multiple lists has little use, in some cases it will be beneficial to include algorithms with a lower individual recommendation quality if they generate recommendations with a different perspective. Most articles targeting recommendations in a carousel setting are evaluated online with users of a certain platform. This puts a high resource requirement on researchers which will limit their ability to investigate this scenario. Furthermore, there seems to lack a standardized evaluation protocol to allow for offline experiments in a carousel setting. This is true, in particular, for how the two-dimensional structure of the user interface is taken into account in ranking metrics for which, to the best of our knowledge, no offline metric exists.

To address the highlighted issues, in this paper we propose a novel offline evaluation protocol that closely mirrors a real user interface with multiple carousels. In this setting, the recommendation quality of each model is computed by how much it improves the accuracy over one or more fixed carousels, in order to better evaluate the user satisfaction in such scenario. The contributions of this paper are as follows:

- We propose an offline evaluation protocol based on real industrial carousel settings and provide experimental results highlighting the different relative accuracy of models evaluated in this way; 
- We extend the widely used NDCG metric [13] to a twodimensional layout, that takes into account the user exploration behavior while navigating carousels. To the best of our knowledge, there exists no ranking metric that takes into consideration a two-dimensional layout.

The rest of the paper is organized as follows. Section 2 presents related works on carousel interfaces. Section 3 provides a description of the carousel scenario and the evaluation protocol. Section 4 reports the results of our experimental analysis. Finally, Section 5 draws conclusions and presents possible future works.

\section{RELATED WORK}

Most of the works targeting a carousel user interface come from industrial research. This demonstrates the importance, at the industrial level, of identifying an adequate combination of carousels to show to the user. However, it may also indicate that the lack of a standardized offline evaluation protocol is hampering researchers who do not have easy access to online evaluation infrastructure, preventing them to work on the topic.

$\mathrm{Wu}$ et al. [18] analyze the problem of optimizing the position of the carousels on the interface of Netflix, a popular online video streaming service. The authors propose a graphical model based on the notion that the benefit of recommending a certain item depends on how novel it is relatively to the previous recommendations, a concept referred to as submodularity. Their algorithm is able to leverage scrolls and navigation feedback to dynamically optimize the user interface. The evaluation is done both online and offline. For the offline evaluation standard metrics are reported (MRR and Precision) considering a carousel as a single item which is relevant if it contains at least a relevant recommendation, therefore not accounting for the ranking within the carousel. Gruson et al. [12] optimize the homepage of Spotify, an online music streaming service. The paper evaluates a series of policies to rank the most relevant playlists for a user. The policies are ranked according to first an online evaluation and then an offline evaluation. The two rankings are then compared and de-biasing techniques are discussed to improve the correspondence of offline evaluations with online ones. In the offline evaluation, the carousels are sequentially concatenated as a single long recommendation list. This evaluation procedure, however, does not take into account the behavior of a user while exploring a two-dimensional interface. Again, the article points out at discrepancies between the online and offline evaluations. Bendada et al. [2] propose a contextual multi-armed bandit online approach to optimize the two-dimensional layout of the homepage of an online music streaming service, called Deezer. Each user is shown a set of carousels which the user can swipe to reveal further items but the system does not log all user actions. To estimate which items the user has seen in the hidden part of the carousels they rely on the cascade model [6] assuming the user has seen all items before the one they interacted with and therefore has swiped and ignored them. The policies are evaluated both online and offline with expected cumulative regrets. Ding et al. [8] targets the problem of whole page optimization for the homepage of Amazon Video, a video streaming service. They assume that a set of carousels is already available and that the objective is to select which carousels to show and in which order while also accounting for the business constraints of the homepage. It should be noted that the personalization of homepage is a widely discussed problem also in other domains. For example, Agarwal et al. [1] propose an optimization framework for the personalized widget layout on Linkedin, a known professional social network site, to improve user engagement. As opposed to the video-on-demand or music streaming scenario where there are many carousels available, in [1] there is only one. Finally, some works try to account for the two-dimensional structure of the page during the algorithm training phase. For example, Elahi and Chandrashekar [9] propose to model the user response to the two-dimensional interface as an embedding.

\section{CAROUSEL EVALUATION CHARACTERISTICS}

In this section we describe the characteristics of a real user interface in an industrial setting using a carousel layout.

Interface: A two-dimensional user interface (i.e., a grid of recommendations) composed of different carousels (i.e., rows). In the general case, carousels may have different lengths which we assume remain constant for all users.

User Behaviour: The user will not explore the interface one carousel at a time, in a sequential way. Rather, they tend to explore a two-dimensional carousel interface focusing on the top-left corner [19] (see also Figure 2).

Recommendations: Each carousel is generated with a different algorithm or may come from a different provider, which may know the general layout of the page and the number of carousels, but will be unaware of the specific recommendations contained in the other carousels ${ }^{1}$. In general, no post-processing step is applied. Hence, the same item may be recommended multiple times across different carousels ${ }^{2}$.

\subsection{Evaluation metrics}

Evaluation in a carousel setting presents broad similarities with a traditional top- $n$ recommendation scenario. An important difference is the presence of duplicates in the recommendation list and the two-dimensional way users explore the interface, which impacts how ranking metrics may consider the item position. In a carousel evaluation scenario, an item in the recommendation list is relevant, i.e., a correct recommendation, if it meets two conditions:

- The item appears in that user's ground truth

- The item has been recommended only once or, if it has duplicates, it is the one corresponding to the best ranking. For traditional top-n ranking with a single recommendation list, duplicates are removed from the list; therefore, we assume that each carousel does not contain duplicates. However, duplicates might occur between carousels. Such duplicates must not be removed during the evaluation, in order to mimic the real behavior of carousel-based user interfaces. Accuracy metrics are not sensitive to the ranking of items; as such,

\footnotetext{
${ }^{1}$ This is common, with content aggregators that aggregate carousels from different providers. For instance, a video content aggregator may have a carousels from Sky, Youtube, Netflix, Prime Video, etc.

${ }^{2}$ For example, in the Netflix homepage shown in Figure 1 the TV series Space Force appears both in the TV Comedies and New Releases carousels.
} 


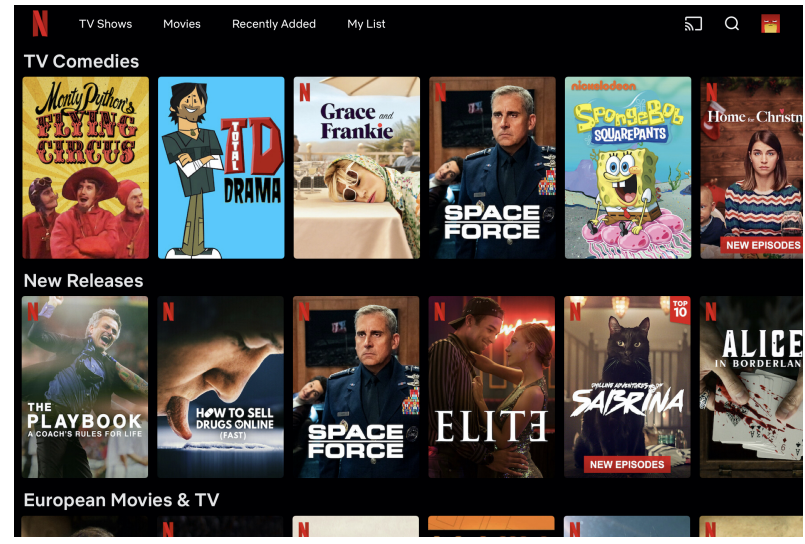

Figure 1: The Netflix homepage, an example of carousel user interface in the multimedia streaming domain.

their measurement with carousel-based used interfaces is the same as with traditional single-list recommender systems. On the other hand, in order to account for the two-dimensional user exploration of the interface a different definition of ranking discount is needed, as described in Section 3.1.1.

3.1.1 Two-dimensional NDCG. The idea of defining a two-dimensional ranking metric stems from the observation that users do not explore each carousel sequentially, but rather start from the top-left corner of the screen and proceed to explore the recommendations both to the right and to the bottom. This behavior has been known for many years in the Information Retrieval field and has been widely researched [4]. Understanding how the user attention varies with more complex interfaces is an active research field but is beyond the scope of this paper. The same phenomenon can be observed for recommender systems with a carousel interface. Figure 2 shows the number of interactions for items displayed in the carousel interface of a video-on-demand service, we can again see how the user interactions are concentrated in the top-left corner [15]. To the best of our knowledge, no ranking metric that takes this behavior into account exists. As a way to approximate this behavior, we propose to extend the commonly used NDCG to the two-dimensional interface. We call this metric NDCG2D, which will weight the item position approximating the two-dimensional position bias.

Traditional NDCG is defined as NDCG $=D C G / I D C G$ where DCG is the Discounted Cumulative Gain computed from the ranking of the relevant items in the recommendation list and IDCG is the Ideal Discounted Cumulative Gain defined as the DCG of the ideal ranked list, i.e., the list composed of all the user's relevant items that can fit in the recommendation list length, ranked according to the ground truth relevance. Given $c$ as the cutoff, i.e., recommendation list length, $\operatorname{rel}(i)$ as the relevance of the item in position $i$, DCG can be computed as shown in Eq. 1.

To account for the two-dimensional position bias, we define as $l$ the number of recommendation lists (i.e., carousels) and extend

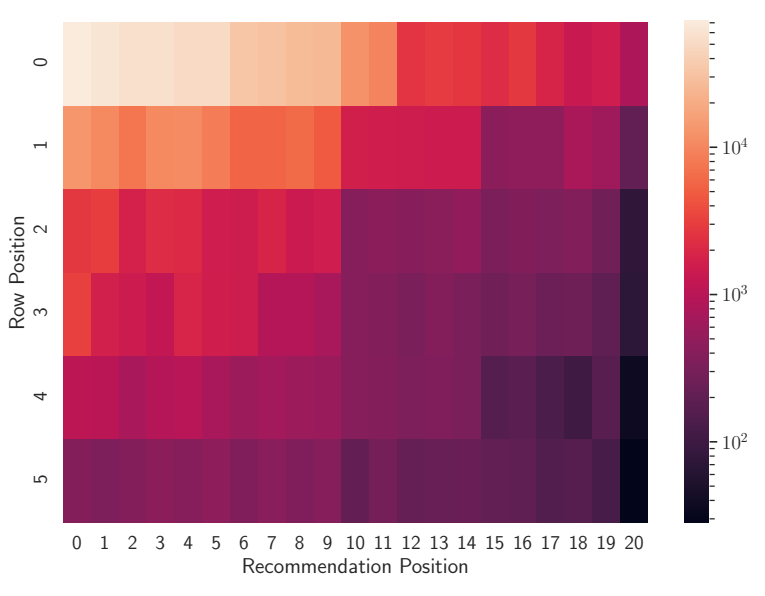

Figure 2: Heatmap of the number of interactions per position on the screen, taken from the dataset presented in [15].

the relevance function to two dimensions as $\operatorname{rel}(i, j)$. The item relevance will be discounted by a quantity proportional to its position in both dimensions. Thus, we define DCG2D as shown in Eq. 2. This metric allows to give importance to both dimensions, according to the weights $(\alpha, \beta \geq 1)$ provided, which can vary depending on the use case. Accordingly, we define its normalized version as $N D C G 2 D=D C G 2 D / I D C G 2 D$. Similarly to NDCG, the IDCG2D will be the DCG2D of the ideal ranking, which is the matrix composed of the user's most relevant items, ranked according to the previously defined two-dimensional position discount, following constraint: for any pair of cells $(i, j),(h, k)$ of the matrix, $\operatorname{rel}(i, j) \geq \operatorname{rel}(h, k)$ if $\alpha i+\beta j<\alpha h+\beta k$. Notice how, in the case of different carousel lengths, the NDCG2D metric can be easily computed by assuming that all the carousels have the maximum carousel length and by treating the missing recommendations at the end of the shorter carousels as simply non-relevant.

\section{EXPERIMENTAL ANALYSIS}

In this section we apply the proposed carousel evaluation on widely used algorithms and compare the results obtained with the traditional evaluation which considers each model independently. We discuss the results of this comparison and highlight some common trends and differences. We release the source code for our experiments in an online repository. ${ }^{3}$

\subsection{Algorithms}

In our evaluation, we included several algorithms developed in the last three decades of research, trying to obtain a broad picture of different families of models.

- Non-Personalized. A simple but effective model, TopPopular recommends to all users the most popular items.

- Nearest-Neighbor Methods. We include in our analysis collaborative filtering (CF) nearest-neighbor techniques such as

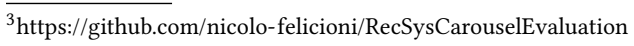




$$
D C G=\sum_{i=1}^{c} \frac{2^{r e l(i)}-1}{\log _{2}(i+1)}
$$

ItemKNN , based on item-item similarities and UserKNN based on user-user similarities. In both cases the similarity is computed with cosine similarity with shrinkage.

- Graph-based Methods. We select two simple approaches that model a random walk in a graph containing user and item nodes. In $\mathbf{P}^{3} \boldsymbol{\alpha}$ [5] the similarity between items is computed as the transition probability between them. $\mathbf{R P}^{3} \boldsymbol{\beta}$ [16] extends $\mathrm{P}^{3} \alpha$ dividing the similarity between two items by their popularity, raised to the power of an additional hyperparameter $\beta$, in order to reduce the popularity bias of the algorithm.

- Content-based and Hybrid Methods. To account for content information we also include content-based models. Among the simplest content based models are neighborhood-based methods that build item-item similarities based on features, ItemKNN-CBF computes the item-based similarity using the item features while UserKNN-CBF computes the userbased similarity using user features. We use the cosine similarity with shrinkage. These methods can be easily extended by creating a new feature vector which concatenates both collaborative and content data. The resulting hybrid algorithms are ItemKNN-CFCBF and UserKNN-CFCBF.

- Machine Learning Approaches. We include several simple but well-known models relying on machine learning, like SLIM ElasticNet (EN) [14], a scalable variant of the original SLIM, and SLIM BPR, a variant of SLIM minimizing the BPR loss. We also include a recent method called EASE $^{R}$ [17], where the author showed how an "embarrassingly shallow" linear model with closed-form solution can outperform much more complex techniques.

- Matrix Factorization Techniques. We include various matrix factorization algorithms, like PureSVD [7], FunkSVD, which are developed for explicit ratings, and iALS, Nonnegative Matrix Factorization (NMF), Matrix Factorization (MF) BPR , focusing on implicit feedback.

\subsection{Hyperparameter optimization}

While in this paper we do not aim to show that any particular model is superior to others, we nonetheless ensure that all algorithms are consistently optimized. To do so we followed the best practices highlighted by Ferrari Dacrema et al. [11] and we relied on the framework published, using a Bayesian search [10] optimizing the MAP metric at cutoff 10 .

\subsection{Datasets}

We report the results for some widely used publicly available datasets. We only selected datasets from domains that tend to use the carousel user interface, in particular video-on-demand. We included MovieLens $10 M$, , a popular dataset of movies recommendations, with 69,878 users, 10,681 items and $10 \mathrm{M}$ ratings. The dataset contains user provided tags for items as well as the year of release and the genre. We conducted experiments also on the Netflix dataset, which is the well known movie dataset from the Netflix Prize. To reduce

$$
D C G 2 D=\sum_{i=1}^{l} \sum_{j=1}^{c} \frac{2^{r e l(i, j)}-1}{\log _{2}(\alpha i+\beta j)}
$$

the computational time, we randomly sampled $20 \%$ of the users. After pre-processing, this dataset has 95,965 users, 17,768 items and almost $20 \mathrm{M}$ ratings. After pre-processing, we randomly selected $80 \%$ of interactions for the training set and $10 \%$ for both validation and test set.

\subsection{Discussion on the results}

For each algorithm, we report both its individual recommendation quality and the evaluation under a carousel setting when the first carousel is fixed and each algorithm is used to fill the second carousel (see Table 1). All recommendation lists have a length, i.e., cutoff, of 10, however note that in the individual evaluation there will be a single recommendation list while in the carousel evaluation there will be more than one, in this case two. We evaluated two cases, in the first one the fixed carousel is a non-personalized TopPopular model, while in the second it is SLIM EN, the personalized model with the highest individual MAP. For the NDCG2D, we chose as an example $\alpha=\beta=1$ in which the user explores both horizontally and vertically with equivalent penalty. The results obtained with the TopPop and SLIM EN fixed carousels and for both datasets are broadly similar. For space reasons we will only discuss the results for MovieLens10M with a SLIM EN fixed carousel (see Table 1). We provide the full results in the online material.

Based on the MAP of the individual evaluation, the best performing algorithms are SLIM, UserKNN and IALS. However, when looking at the results of the carousel evaluation, we can see that many differences emerge. For example, $\mathrm{P}^{3} \alpha$ has an individual MAP that is $39.5 \%$ lower than SLIM EN, while $\mathrm{EASE}^{R}$ only $11.5 \%$ lower. In the carousel evaluation instead, the improvement provided by these two algorithms is similar with $\mathrm{P}^{3} \alpha$ being slightly better. The discrepancy between individual and carousel evaluation is even clearer if we look at the column showing the difference in the rankings of all algorithms in the two scenarios. As a general trend we can see that the relative performance of the models differ, resulting in several changes to the ranking of the algorithms. Some models, in this case all matrix factorization algorithms (except for PureSVD) gain several positions. NMF and MF BPR jump up by 6 positions. On the other hand, item-based machine learning models tend to lose some positions. As a result, in the carousel evaluation the best algorithms are IALS, FunkSVD and UserKNN. The difference in those rankings lies in how those recommendations intersect. Algorithms which will tend to recommend items similar to the ones provided by SLIM EN will be penalized in this carousel evaluation, whereas algorithms providing accurate but different recommendations will be advantaged. An interesting case is $\mathrm{EASE}^{R}$, which loses 8 positions, probably because of its resemblance with the SLIM algorithm.

Regarding the NDCG2D metric, we can notice how it too can lead to different decisions with respect to the standard NDCG in the carousel evaluation, though to a lesser extent. Looking at the results in Table 1, we notice that the NDCG results of $\mathrm{EASE}^{R}$ and Item KNN are very similar under the carousel evaluation, but NDCG2D will 


\begin{tabular}{|c|c|c|c|c|c|c|c|c|c|c|c|c|}
\hline & \multicolumn{3}{|c|}{ Individual } & \multicolumn{4}{|c|}{ Carousel (SLIM EN) } & \multicolumn{2}{|c|}{ Improvement on SLIM EN } & \multicolumn{3}{|c|}{ MAP rank } \\
\hline & PREC & MAP & NDCG & PREC & MAP & NDCG & NDCG 2D & Individual & Carousel & Individual & Carousel & $\Delta$ rank \\
\hline SLIM EN & 0.2460 & 0.2340 & 0.2856 & - & - & - & - & - & - & - & - & - \\
\hline TopPop & 0.0975 & 0.0709 & 0.0983 & $\mid 0.1399$ & 0.1895 & 0.2967 & 0.2939 & $-69.7 \%$ & $+4.8 \%$ & 13 & 13 & 0 \\
\hline UserKNN CF & 0.2343 & 0.2251 & 0.2815 & 0.1528 & 0.1955 & 0.3137 & 0.3225 & $-3.8 \%$ & $+8.1 \%$ & 1 & 3 & -2 \\
\hline ItemKNN CF & 0.1885 & 0.1728 & 0.2122 & 0.1455 & 0.1921 & 0.3015 & 0.3034 & $-26.2 \%$ & $+6.3 \%$ & 8 & 9 & -1 \\
\hline $\mathrm{P}^{3} \alpha$ & 0.1646 & 0.1414 & 0.1915 & 0.1433 & 0.1912 & 0.3009 & 0.3021 & $-39.6 \%$ & $+5.7 \%$ & 12 & 10 & +2 \\
\hline $\mathrm{RP}^{3} \beta$ & 0.1886 & 0.1686 & 0.2160 & 0.1430 & 0.1908 & 0.3014 & 0.3026 & $-28.0 \%$ & $+5.5 \%$ & 9 & 11 & -2 \\
\hline EASE $^{R}$ & 0.2260 & 0.2070 & 0.2566 & 0.1430 & 0.1899 & 0.3017 & 0.3012 & $-11.5 \%$ & $+5.1 \%$ & 4 & 12 & -8 \\
\hline SLIM BPR & 0.2274 & 0.2159 & 0.2699 & 0.1490 & 0.1937 & 0.3084 & 0.3138 & $-7.7 \%$ & $+7.2 \%$ & 2 & 6 & -4 \\
\hline MF BPR & 0.1759 & 0.1502 & 0.1882 & 0.1479 & 0.1937 & 0.3040 & 0.3069 & $-35.8 \%$ & $+7.2 \%$ & 11 & 5 & +6 \\
\hline MF FunkSVD & 0.2039 & 0.1748 & 0.2307 & 0.1560 & 0.1979 & 0.3148 & 0.3248 & $-25.3 \%$ & $+9.5 \%$ & 7 & 2 & +5 \\
\hline PureSVD & 0.2217 & 0.2060 & 0.2527 & 0.1471 & 0.1924 & 0.3039 & 0.3061 & $-12.0 \%$ & $+6.4 \%$ & 5 & 7 & -2 \\
\hline NMF & 0.1872 & 0.1613 & 0.1974 & 0.1484 & 0.1938 & 0.3037 & 0.3064 & $-31.1 \%$ & $+7.2 \%$ & 10 & 4 & +6 \\
\hline IALS & 0.2329 & 0.2152 & 0.2539 & 0.1592 & 0.1998 & 0.3101 & 0.3174 & $-8.1 \%$ & $+10.5 \%$ & 3 & 1 & +2 \\
\hline ItemKNN CBF & 0.0113 & 0.0052 & 0.0079 & $\mid 0.1264$ & 0.1826 & 0.2875 & 0.2765 & $-97.8 \%$ & $+1.0 \%$ & 14 & 14 & 0 \\
\hline ItemKNN CFCBF & 0.1952 & 0.1790 & 0.2174 & $\mid$\begin{tabular}{|l|}
0.1460 \\
$\mid$
\end{tabular} & 0.1923 & 0.3021 & 0.3044 & $-23.5 \%$ & $+6.4 \%$ & 6 & 8 & -2 \\
\hline & & & & Indiv & dual & & $\mathrm{Car}$ & usel & & & & \\
\hline & & & & valuated & algorithm & @10 & & $E N$ & @10 & & & \\
\hline & & & & & & & Evalua & 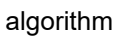 & {$[0$} & & & \\
\hline
\end{tabular}

Table 1: Comparison of the MovieLens10M accuracy metrics with individual and carousel evaluation (with SLIM EN fixed as the first carousel) at recommendation list length of 10 . Note that in the carousel evaluation there will be two recommendation lists. The improvement over the SLIM EN carousel is computed with MAP.

give a slight preference for Item $\mathrm{KNN}$ instead. This difference will become more marked as the page layout becomes more complex including more and longer carousels.

\section{CONCLUSIONS AND FUTURE WORK}

This paper proposes a new offline evaluation protocol for a carousel user interface, where the recommendation quality of a model is not measured independently but rather is put into the context of other recommendation lists being already available to the users. The experimental analysis shows that the relative ranking of the personalized algorithms changes when a carousel is fixed as the first displayed to the user. This is in line with previous observations that the correlations between models have an important role to play and should be taken into account during offline evaluation as well. Further study is needed to better understand this impact. Important future work is an online study to measure how closely the offline carousel evaluation is able to represent the user behavior, as well as extended offline evaluation, involving a wider array of scenarios, for example sequential recommendation and also including families of models not analyzed in this paper.

Ultimately, the carousel evaluation protocol will allow researchers to conduct offline evaluations in these industrially relevant scenarios and open a wide number of research possibilities in studying how to combine the strength of various models and techniques to provide the user with ever more accurate and interesting recommendations.

\section{REFERENCES}

[1] Deepak Agarwal, Shaunak Chatterjee, Yang Yang, and Liang Zhang. 2015. Constrained Optimization for Homepage Relevance. In Proceedings of the 24th International Conference on World Wide Web Companion, WWW 2015, Florence, Italy, May 18-22, 2015 - Companion Volume, Aldo Gangemi, Stefano Leonardi, and Alessandro Panconesi (Eds.). ACM, 375-384. https://doi.org/10.1145/2740908.2745398

[2] Walid Bendada, Guillaume Salha, and Théo Bontempelli. 2020. Carousel Personalization in Music Streaming Apps with Contextual Bandits. In RecSys 2020: Fourteenth ACM Conference on Recommender Systems, Virtual Event, Brazil, September 22-26, 2020, Rodrygo L. T. Santos, Leandro Balby Marinho, Elizabeth M. Daly, Li Chen, Kim Falk, Noam Koenigstein, and Edleno Silva de Moura (Eds.). ACM, 420-425. https://doi.org/10.1145/3383313.3412217

[3] Laming Chen, Guoxin Zhang, and Eric Zhou. 2018. Fast greedy map inference for determinantal point process to improve recommendation diversity. In Advances in Neural Information Processing Systems. 5622-5633.

[4] Flavio Chierichetti, Ravi Kumar, and Prabhakar Raghavan. 2011. Optimizing twodimensional search results presentation. In Proceedings of the Forth International Conference on Web Search and Web Data Mining, WSDM 2011, Hong Kong, China, February 9-12, 2011, Irwin King, Wolfgang Nejdl, and Hang Li (Eds.). ACM, 257266. https://doi.org/10.1145/1935826.1935873

[5] Colin Cooper, Sang-Hyuk Lee, Tomasz Radzik, and Yiannis Siantos. 2014. Random walks in recommender systems: exact computation and simulations. In 23rd International World Wide Web Conference, WWW'14, Seoul, Republic of Korea, April 7-11, 2014, Companion Volume, Chin-Wan Chung, Andrei Z. Broder, Kyuseok Shim, and Torsten Suel (Eds.). ACM, 811-816. https://doi.org/10.1145/2567948. 2579244

[6] Nick Craswell, Onno Zoeter, Michael J. Taylor, and Bill Ramsey. 2008. An experimental comparison of click position-bias models. In Proceedings of the International Conference on Web Search and Web Data Mining, WSDM 2008, Palo Alto, California, USA, February 11-12, 2008, Marc Najork, Andrei Z. Broder, and Soumen Chakrabarti (Eds.). ACM, 87-94. https://doi.org/10.1145/1341531.1341545

[7] Paolo Cremonesi, Yehuda Koren, and Roberto Turrin. 2010. Performance of recommender algorithms on top-n recommendation tasks. In Proceedings of the 2010 ACM Conference on Recommender Systems, RecSys 2010, Barcelona, Spain, September 26-30, 2010, Xavier Amatriain, Marc Torrens, Paul Resnick, and Markus Zanker (Eds.). ACM, 39-46. https://doi.org/10.1145/1864708.1864721

[8] Weicong Ding, Dinesh Govindaraj, and S. V. N. Vishwanathan. 2019. Whole Page Optimization with Global Constraints. In Proceedings of the 25th ACM 
SIGKDD International Conference on Knowledge Discovery \& Data Mining, KDD 2019, Anchorage, AK, USA, August 4-8, 2019, Ankur Teredesai, Vipin Kumar, Ying Li, Rómer Rosales, Evimaria Terzi, and George Karypis (Eds.). ACM, 3153-3161. https://doi.org/10.1145/3292500.3330675

[9] Ehtsham Elahi and Ashok Chandrashekar. 2020. Learning Representations of Hierarchical Slates in Collaborative Filtering. In RecSys 2020: Fourteenth ACM Conference on Recommender Systems, Virtual Event, Brazil, September 22-26, 2020 Rodrygo L. T. Santos, Leandro Balby Marinho, Elizabeth M. Daly, Li Chen, Kim Falk, Noam Koenigstein, and Edleno Silva de Moura (Eds.). ACM, 703-707. https: //doi.org/10.1145/3383313.3418484

[10] Nicolò Felicioni, Andrea Donati, Luca Conterio, Luca Bartoccioni, Davide Yi Xian $\mathrm{Hu}$, Cesare Bernardis, and Maurizio Ferrari Dacrema. 2020. Multi-Objective Blended Ensemble For Highly Imbalanced Sequence Aware Tweet Engagement Prediction. In RecSys Challenge '20: Proceedings of the Recommender Systems Challenge 2020, Virtual Event Brazil, September, 2020. ACM, 29-33. https://dl.acm. org/doi/10.1145/3415959.3415998

[11] Maurizio Ferrari Dacrema, Simone Boglio, Paolo Cremonesi, and Dietmar Jannach. 2021. A Troubling Analysis of Reproducibility and Progress in Recommende Systems Research. ACM Trans. Inf. Syst. 39, 2, Article 20 (Jan. 2021), 49 pages. https://doi.org/10.1145/3434185

[12] Alois Gruson, Praveen Chandar, Christophe Charbuillet, James McInerney, Samantha Hansen, Damien Tardieu, and Ben Carterette. 2019. Offline Evaluation to Make Decisions About PlaylistRecommendation Algorithms. In Proceedings of the Twelfth ACM International Conference on Web Search and Data Mining, WSDM 2019, Melbourne, VIC, Australia, February 11-15, 2019, J. Shane Culpepper, Alistair Moffat, Paul N. Bennett, and Kristina Lerman (Eds.). ACM, 420-428. https://doi.org/10.1145/3289600.3291027

[13] Kalervo Järvelin and Jaana Kekäläinen. 2000. IR evaluation methods for retrieving highly relevant documents. In SIGIR 2000: Proceedings of the 23rd Annual International ACM SIGIR Conference on Research and Development in Information Retrieval, fuly 24-28, 2000, Athens, Greece, Emmanuel J. Yannakoudakis,
Nicholas J. Belkin, Peter Ingwersen, and Mun-Kew Leong (Eds.). ACM, 41-48. https://doi.org/10.1145/345508.345545

[14] Mark Levy and Kris Jack. 2013. Efficient top-n recommendation by linear regression. In RecSys Large Scale Recommender Systems Workshop.

[15] Fernando Benjamín Pérez Maurera, Maurizio Ferrari Dacrema, Lorenzo Saule, Mario Scriminaci, and Paolo Cremonesi. 2020. ContentWise Impressions: An Industrial Dataset with Impressions Included. In CIKM '20: The 29th ACM International Conference on Information and Knowledge Management, Virtual Event, Ireland, October 19-23, 2020, Mathieu d'Aquin, Stefan Dietze, Claudia Hauff, Edward Curry, and Philippe Cudré-Mauroux (Eds.). ACM, 3093-3100. https://doi.org/10.1145/3340531.3412774

[16] Bibek Paudel, Fabian Christoffel, Chris Newell, and Abraham Bernstein. 2017. Updatable, Accurate, Diverse, and Scalable Recommendations for Interactive Applications. ACM Trans. Interact. Intell. Syst. 7, 1 (2017), 1:1-1:34. https: //doi.org/10.1145/2955101

[17] Harald Steck. 2019. Embarrassingly Shallow Autoencoders for Sparse Data. In The World Wide Web Conference, WWW 2019, San Francisco, CA, USA, May 1317, 2019, Ling Liu, Ryen W. White, Amin Mantrach, Fabrizio Silvestri, Julian J. McAuley, Ricardo Baeza-Yates, and Leila Zia (Eds.). ACM, 3251-3257. https: //doi.org/10.1145/3308558.3313710

[18] Chao-Yuan Wu, Christopher V. Alvino, Alexander J. Smola, and Justin Basilico. 2016. Using Navigation to Improve Recommendations in Real-Time. In Proceedings of the 10th ACM Conference on Recommender Systems, Boston, MA, USA, September 15-19, 2016, Shilad Sen, Werner Geyer, Jill Freyne, and Pablo Castells (Eds.). ACM, 341-348. https://doi.org/10.1145/2959100.2959174

[19] Qian Zhao, Shuo Chang, F. Maxwell Harper, and Joseph A. Konstan. 2016. Gaze Prediction for Recommender Systems. In Proceedings of the 10th ACM Conference on Recommender Systems, Boston, MA, USA, September 15-19, 2016, Shilad Sen, Werner Geyer, Jill Freyne, and Pablo Castells (Eds.). ACM, 131-138. https: //doi.org/10.1145/2959100.2959150 